\title{
Siberia at the Last Glacial Maximum: Environment and Archaeology
}

\section{Yaroslav V. Kuzmin}

\section{Erratum to: J Archaeol Res DOI 10.1007/s10814-007-9019-6}

In Table 5, the column heading "Ust-Kova" should be "Ust-Kova (middle complex)." The + symbol used in the last column indicates that the respective tools were present, but no numbers were given.

The online version of the original article can be found under doi:10.1007/s10814-007-9019-6. 